\title{
A Case of Accidental Vincristine Overdose and Effective use of Therapeutic Plasma Exchange in Its Management
}

\begin{abstract}
Vincristine is a common drug used in hematological malignancy with a maximum per dose limited to $2 \mathrm{mg}$ mainly to limit neurotoxicity. Vincristine overdose and wrong route of administration, though has been reported in world literature, but its appropriate management is still undefined. We report a case of Hodgkin lymphoma being treated with adriamycin, bleomycin, vinblastine and dacarbazine protocol, where vincristine was accidently administrated instead of vinblastine due to its "look-alike, sound-alike" nature. The patient developed severe toxicity due to the same, which was effectively managed by therapeutic plasma exchange.
\end{abstract}

Keywords: Look-alike, sound-alike drugs, medication error, overdose, therapeutic plasma exchange, vincristine

\section{Introduction}

Vincristine is an antimicrotubular agent belonging to vinca alkaloid subtype of antineoplastic drugs. The common adverse effects of it are alopecia, constipation, myelosuppression, and neuropathy. Other drugs belonging to the same subtype of antineoplastic drugs are Vinblastine, Vindesine and Vinorelbine. Due to this subtype of antineoplastic drugs being "Look-alike, sound-alike" (LASA) drugs and part of various protocols of haematological malignancies, there are high chances of human errors occurring during administration of these drugs. Despite high chances of human error, only a limited number of case reports have been published in literature about wrong doses, route of administration, with the adverse effects and management of the same.

Vincristine overdose and wrong route of administration though has been reported in world literature and their appropriate management is still undefined. Till date, from India only one case report of the wrong route of administration with no report of vincristine overdose has been published despite vincristine being a common drug used in haematological malignancies. ${ }^{[1]}$ We present here the first reported case of vincristine overdose from

This is an open access journal, and articles are distributed under the terms of the Creative Commons Attribution-NonCommercial-ShareAlike 4.0 License, which allows others to remix, tweak, and build upon the work non-commercially, as long as appropriate credit is given and the new creations are licensed under the identical terms.

For reprints contact: WKHLRPMedknow_reprints@wolterskluwer.com
India with effective use of therapeutic plasma exchange (TPE) to combat its toxicity.

\section{Case Report}

An 18-year-old male, a recently diagnosed case of Hodgkin lymphoma, was admitted for doxorubicin (adriamycin), bleomycin, vinblastine and dacarbazine (ABVD) protocol. During the first cycle day 1 vincristine $9 \mathrm{mg}\left(6 \mathrm{mg} / \mathrm{m}^{2}\right)$ was accidently administrated instead of vinblastine. The mistake was immediately identified and the patient was then immediately started on folinic acid $100 \mathrm{mg}$ intravenous every $3 \mathrm{~h}$ for the first $24 \mathrm{~h}$ and then every $6 \mathrm{~h}$, prokinetics with laxatives, oral care with betadine mouthwash and gargles, intravenous fluids with sodium chloride $(0.9 \%)$, vitamin B-complex and other supportive measures. Despite above severe oral mucositis, generalized weakness, obstipation, nausea, loss of appetite, weight loss (4 $\mathrm{kg}$ on day 4), reticent behaviour with episodes of restlessness in between, hyponatremia (syndrome of inappropriate antidiuretic hormone secretion) from day 2-3 which increased with time. His laboratory parameters on day 4 were (before TPE): haemoglobin - $11.9 \mathrm{~g} / \mathrm{dl}$, platelet count - $2.8 \mathrm{lakh} / \mu \mathrm{L}$, total leukocyte count - 12,400/ $\mu \mathrm{L}$ (neutrophils: 91\%,

\footnotetext{
How to cite this article: Goyal $\mathrm{H}$, Khetarpal $\mathrm{A}$, Jain AK. A case of accidental vincristine overdose and effective use of therapeutic plasma exchange in its management. Indian $\mathrm{J}$ Med Paediatr Oncol 2020;41:770-2.
} measures, the patient had severe jaw pain,

\author{
Hari Goyal' ${ }^{1}$, \\ Anil Khetarpal'2, \\ Amit Kumar Jain ${ }^{1}$ \\ ${ }^{I}$ Department of Medical \\ Oncology, Artemis Hospital, \\ Gurugram, Haryana, India, \\ ${ }^{2}$ Department of Blood Bank and \\ Transfusion Medicine, Artemis \\ Hospital, Gurugram, \\ Haryana, India
}

Submitted: 30 -Mar-2020 Revised: 27-May-2020 Accepted: 29-May-2020 Published: 29-Oct-2020

\footnotetext{
Address for correspondence:

Dr. Amit Kumar Jain, Department of Medical Oncology, Artemis Hospital, Sector-51, Gurugram - 122 001, Haryana, India.

E-mail:20akjain@gmail.com
}

Access this article online Website: www.ijmpo.org

DOI: 10.4103/ijmpo.ijmpo_116_20 Quick Response Code:

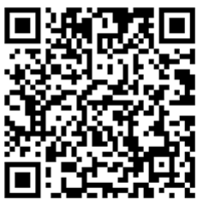


lymphocytes: $8 \%$ ), sodium - $124 \mathrm{mEq} / \mathrm{L}$, potassium - $3.8 \mathrm{mEq} / \mathrm{L}$, creatinine $-0.55 \mathrm{mg} / \mathrm{dl}$, total bilirubin - $0.3 \mathrm{mg} / \mathrm{dl}$, serum glutamic oxaloacetic transaminase (SGOT) - 24 IU/L, serum glutamic pyruvic transaminase (SGPT) - $38 \mathrm{IU} / \mathrm{L}$, alkaline phosphatase - $110 \mathrm{IU} / \mathrm{L}$, albumin - $3.2 \mathrm{~g} / \mathrm{dl}$. After a thorough literature search and discussing patient's deteriorating condition with the patient's attendants, a decision of TPE was taken and done on day 4 after obtaining an informed consent from the patient's attendant.

TPE was performed with Fresenius COM. TEC apheresis device using right femoral double lumen haemodialysis catheter as venous access. Patient was premedicated with hydrocortisone $100 \mathrm{mg}$ and pheniramine $22.75 \mathrm{mg}$. One volume $(2200 \mathrm{ml})$ of plasma was $100 \%$ exchanged with $4 \%$ albumin $(1500 \mathrm{ml})$ and fresh frozen plasma $(700 \mathrm{ml})$ along with continuous calcium gluconate infusion $(1 \mathrm{~g}$ in $200 \mathrm{ml}$ normal saline). It was a 2 -h uneventful procedure. The patient started to show improvement from his toxic symptoms which were evident post $90 \mathrm{~min}$ of starting TPE with dramatic improvement from his toxic symptoms post completion of TPE. The patient was then monitored and discharged on day 7 with resolution of all his toxic symptoms. On day 7 his laboratory parameters were: haemoglobin - $12.2 \mathrm{~g} / \mathrm{dl}$, platelet count - 4.6 lakh $/ \mu \mathrm{L}$, total leukocyte count - 5900/ $\mu \mathrm{L}$ (neutrophils: 69\%, lymphocytes: 26\%), sodium - $132 \mathrm{mEq} / \mathrm{L}$, potassium - $3.8 \mathrm{mEq} / \mathrm{L}$, creatinine - $0.61 \mathrm{mg} / \mathrm{dl}$, total bilirubin - $0.4 \mathrm{mg} / \mathrm{dl}$, SGOT - $23 \mathrm{IU} / \mathrm{L}, \mathrm{SGPT}-27 \mathrm{IU} / \mathrm{L}$, alkaline phosphatase - $85 \mathrm{IU} / \mathrm{L}$, albumin - $4.1 \mathrm{~g} / \mathrm{dl}$. He then completed all his 6 cycles of ABVD uneventfully and was in complete metabolic response on completion of his therapy 6 months back. He is now on regular 3 monthly follow-up since then with no active complaints, clinically normal, routine laboratory test within normal limits and has no features of any long-term toxicity.

\section{Discussion}

Vincristine, an antimicrotubular agent, belonging to vinca alkaloid subtype of antineoplastic drugs is a common drug used in oncology especially in haematological malignancy. With many approved and off-label indications it is only administrated intravenously with usual dose varying between 0.5 and $1.4 \mathrm{mg} / \mathrm{m}^{2}$ with frequency and dosing based on the protocol and treatment phase. Its maximum per dose is limited to $2 \mathrm{mg}$ mainly to limit neurotoxicity. The common adverse effects of it being alopecia, constipation, myelosuppression and neuropathy. There is no specific antidote for overdose but folinic acid and TPE is recommended, based on anecdotal experience and treatment is mainly supportive to alleviate its adverse effects predominantly that of neurotoxicity, myelosuppression and ileus. $^{[2,3]}$

Other drugs belonging to same subtype of antineoplastic drugs are Vinblastine, Vindesine and Vinorelbine. Due to this subtype, antineoplastic drugs being LASA drugs and part of various protocols of haematological malignancies, chances of human error during administration of these drugs are high.

Though an accidently overdosing due to LASA drug resulted in toxicity our case; it adds to the few case reports in the literature supporting use of TPE in vincristine overdose. Overdose of drugs is American Society for Apheresis category II indication for TPE. ${ }^{[4]}$ It is indicated in drugs with low volume of distribution, high protein binding and long half-life. ${ }^{[5]}$ Vincristine has a high terminal phase of elimination especially in higher dose thus a suitable target for TPE in overdose toxicity. ${ }^{[6]}$

We also learn from this case that medication errors should be prevented, especially with respect to antineoplastic drugs as these errors can be potentially life-threatening. It also emphasizes the need of implementation for medication safety practices, with a few important ones being listed below.

- Double cross verification of drugs (including prescription error), dose (including decimal errors) and its administration method

- Computerized or printed orders with use of tall-man lettering for LASA drug names

- Regulating dispensing of drugs

- Instant access to chemotherapy protocols and drug information by the staff

- Administration of drug especially, chemotherapy only by a trained and experienced staff attending regular continuous medical education

- Avoid initiation and/or administration of chemotherapy during night

- Medication error reporting system.

The root cause analysis of the overdosing in our case found that the medication error mainly due to similar sounding names of the drug and required stricter implementation of medication safety practices to prevent or minimize any medication error in the future.

\section{Conclusion}

This case adds to the few case reports in the literature supporting use of TPE in vincristine overdose. It also emphasizes the importance of implementation medication safety practices in the hospital, as medication errors can sometimes lead to devastating consequences like permanent damage and death.

\section{Declaration of patient consent}

The authors certify that they have obtained all appropriate patient consent forms. In the form, the patient has given his consent for his images and other clinical information to be reported in the journal. The patient understand that name and initials will not be published and due efforts will be made to conceal identity, but anonymity cannot be guaranteed. 


\section{Financial support and sponsorship}

Nil.

\section{Conflicts of interest}

There are no conflict of interest.

\section{References}

1. Saha AS, Islam MF, Bhattacharya S, Giri PP. Clinical presentation of inadvertent intrathecal vincristine masquerading guillain-barre syndrome. Indian J Hematol Blood Transfus 2016;32:59-61.

2. Chae L, Moon HS, Kim SC. Overdose of vincristine: Experience with a patient. J Korean Med Sci 1998;13:334-8.

3. Kosmidis HV, Bouhoutsou DO, Varvoutsi MC, Papadatos J,
Stefanidis CG, Vlachos P, et al. Vincristine overdose: Experience with 3 patients. Pediatr Hematol Oncol 1991;8:171-8.

4. Schwartz J, Padmanabhan A, Aqui N, Balogun RA, Connelly-Smith L, Delaney $\mathrm{M}$, et al. Guidelines on the use of therapeutic apheresis in clinical practice-evidence-based approach from the writing committee of the American Society for Apheresis: The Seventh Special Issue. J Clin Apheresis 2016;31:149-62.

5. Ibrahim RB, Balogun RA. Medications and therapeutic apheresis procedures: Are we doing our best? J Clin Apher 2013;28:73-7.

6. Estlin EJ, Ronghe M, Burke GA, Yule SM. The clinical and cellular pharmacology of vincristine, corticosteroids, 1-asparaginase, anthracyclines and cyclophosphamide in relation to childhood acute lymphoblastic leukaemia. Br J Haematol 2000;110:780-90. 